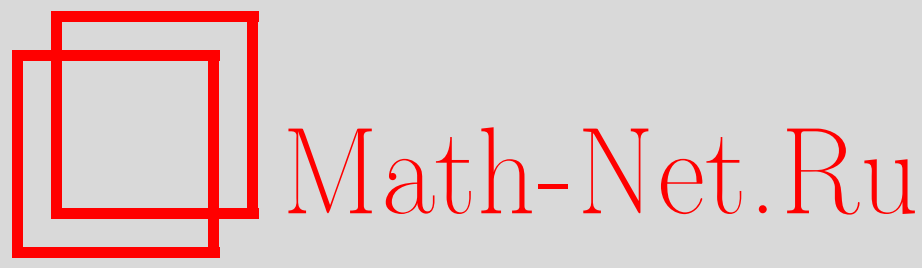

В. Р. Халилов, Ки Ын Ли, Дискретные спектры дираковского гамильтониана в кулоновских потенциалах и потенциалах Ааронова-Бома в $2+1$ измерениях, ТМФ, 2011, том 169, номер 3, 368-390

DOI: https://doi.org/10.4213/tmf6736

Использование Общероссийского математического портала Math-Net.Ru подразумевает, что вы прочитали и согласны с пользовательским соглашением http: //www . mathnet.ru/rus/agreement

Параметры загрузки:

IP : 54.147 .182 .235

26 апреля 2023 г., $16: 26: 32$

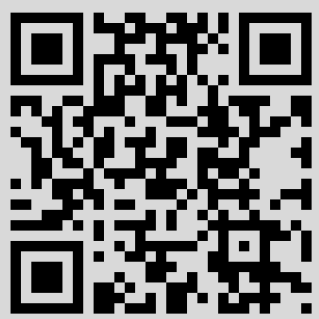




\title{
ФИЗИКА
}

Том 169, № 3

декабрь, 2011

\section{ДИСКРЕТНЫЕ СПЕКТРЫ ДИРАКОВСКОГО ГАМИЛЬТОНИАНА В КУЛОНОВСКИХ ПОТЕНЦИАЛАХ И ПОТЕНЦИАЛАХ ААРОНОВА-БОМА В $2+1$ ИЗМЕРЕНИЯХ}

\begin{abstract}
Найдены все самосопряженные дираковские гамильтонианы в кулоновских потенциалах и потенциалах Ааронова-Бома в $2+1$ измерениях с учетом спина фермиона. Получены уравнения, неявно определяющие спектры, и построены собственные функции для всех самосопряженных дираковских гамильтонианов в указанных внешних полях. В некоторых случаях решения уравнений для спектров найдены явно.
\end{abstract}

Ключевые слова: симметрический оператор, самосопряженные расширения гамильтониана, кулоновский потенциал в $2+1$ измерениях, потенциал Ааронова-Бома, спин.

\section{1. ВВЕДЕНИЕ}

Интерес к физическим явлениям в квантовых системах релятивистских фермионов в присутствии интенсивных внешних полей в пространствах пониженных размерностей, проявляемый в последние годы, вызван открытием ряда эффектов физики конденсированных сред [1], возможностью применения полученных для этих моделей результатов для изучения эффекта Ааронова-Бома (АБ) [2], квантового эффекта Холла [3], высокотемпературной сверхпроводимости [4], [5], а также физических процессов в присутствии космических струн [6]-[8]. В частности, поведение фермионов в поле космической струны можно описать решениями уравнения Дирака в потенциале АБ в $2+1$ измерениях [6]. Решения уравнения Дирака для фермионов нулевой массы в $2+1$ измерениях описывают состояния фермионов в графене [9]-[11].

Известно [12], что гамильтониан в уравнении Шредингера с потенциалом АБ сингулярен и поэтому требует дополнительного доопределения для того, чтобы его можно было трактовать как самосопряженный квантово-механический оператор. Для этого необходимо указать область его определения в гильбертовом пространстве квадратично-интегрируемых функций. Впервые самосопряженный гамильтониан

* Московский государственный университет им. М.В. Ломоносова, Москва, Россия. E-mail: khalilov@phys.msu.ru, skyangel26@gmail.com. 
для этой задачи был построен в работе [12]. В большинстве физически интересных задач квантовой механики гамильтонианы являются сингулярными операторами, а в естественных областях определения - только симметрическими операторами. Поэтому возникает задача построения самосопряженного гамильтониана во всей области определения. Для решения этой задачи нужно найти все самосопряженные расширения данного симметрического оператора и затем выделить корректный самосопряженный гамильтониан с помощью физически приемлемых граничных условий в точке сингулярности гамильтониана.

Впервые задача построения самосопряженного дираковского гамильтониана в потенциале АБ в 2+1 измерениях была решена в работах [7], [8]. Так, в работе [7] было показано, что область определения самосопряженного расширения гамильтониана наряду с регулярными может содержать сингулярные квадратично-интегрируемые при $r=0$ функции, а также было найдено формальное решение, описывающее связанное состояние фермиона в поле космической струны.

Другой интересный с точки зрения физики случай сингулярного гамильтониана дираковский гамильтониан в сильном кулоновском поле точечного заряда (источника). Построение самосопряженного гамильтониана в этом поле во всей области позволит ответить на вопрос о стабильности вакуума квантовой электродинамики (КЭД) в присутствии сильного кулоновского поля. Важнейший вопрос о стабильности вакуума был всесторонне изучен в многочисленных работах (см. работы [13]-[17] и ссылки в них) в $3+1$ измерениях и в работах [18], [19] - в $2+1$ измерениях. Во всех этих работах вместо точечного источника рассматривался кулоновский потенциал, обрезанный на малом расстоянии $R$ от источника, что фактически эквивалентно постановке граничного условия в точке $R$. Физически постановка такого граничного условия означает учет конечных размеров источника поля. Такой способ определения самосопряженного гамильтониана называют физической регуляризацией [12].

Напомним, что энергия электрона в основном состоянии в кулоновском поле, заданном 4-вектор-потенциалом $A^{0}(r)=-a /\left(e_{0} r\right), \mathbf{A}=0, a>0\left(-e_{0}<0\right.$ - заряд электрона),

$$
E_{g}=m \sqrt{1-a^{2}}
$$

обращается в нуль при $a=1$, а при $a>1$ интерпретация этой формулы как энергии электрона вообще теряет смысл. В кулоновском потенциале, обрезанном на малом расстоянии $R$, низший уровень энергии электрона становится отрицательным при $a>1$ и при дальнейшем увеличении $a$ может достичь границы нижнего континуума (отрицательных) энергий $-m$, а значение $a=a_{\mathrm{c}}$, при котором низший уровень энергии электрона равен $-m$, называют критическим зарядом для основного состояния.

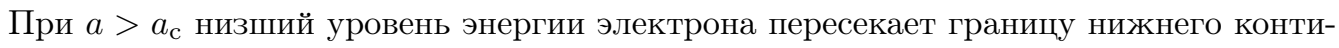
нуума энергий, а вакуум КЭД в сильном кулоновском потенциале перестраивается так, что в вакуумной оболочке кулоновского центра появляется новое состояние с энергией $E<-m$ и проекцией спина $s= \pm 1$. С точки зрения КЭД вакуум при $a>a_{\text {c }}$ становится неустойчивым, что приводит к рождению позитронов, и одновременно приобретает отрицательный электрический заряд, который, очевидно, будет равен двум зарядам электрона [13]-[17]. В задаче о поведении электрона в сильном (обрезанном) кулоновском поле в $2+1$ измерениях картина похожа, но энергия электрона в основном состоянии становится равной нулю при $a=1 / 2$ [18], [19]. 
Впервые самосопряженные дираковские гамильтонианы в сильном кулоновском поле точечного источника были построены в работе [20].

В работе [19] установлено, что радиальные гамильтонианы в кулоновском поле точечного источника в $3+1$ измерениях и в кулоновских и АБ-потенциалах в $2+1$ измерениях фактически одинаковы за исключением того, что гамильтониан в $2+1$ измерениях содержит параметр, характеризующий спин фермиона, явно. Это позволяет изучить влияние спина частицы на релятивистские связанные состояния фермиона в двумерных кулоновских полях и в потенциале АБ. Здесь нелишне напомнить, что решения уравнения Дирака в $2+1$ измерениях представляют собой спинор, верхняя и нижняя компоненты которого интерпретируются соответственно как положительно- и отрицательно-частотные состояния. Для описания спина фермиона в двухкомпонентное уравнение Дирака в потенциале АБ вводится спиновый параметр.

В настоящей статье мы представляем математически строгое квантово-механическое описание движения фермиона в двумерных кулоновских (векторном и скалярном) полях и в потенциале АБ. Для этого мы находим все самосопряженные дираковские гамильтонианы в кулоновских и АБ-потенциалах в $2+1$ измерениях с учетом спина фермиона. Затем мы получаем уравнения, неявно определяющие спектры дираковских гамильтонианов в указанных внешних полях, и обсуждаем вопрос о собственных функциях всех самосопряженных гамильтонианов. Решение этих задач проводится методами теории самосопряженных расширений (фон Неймана) симметрических операторов и направляющих функционалов Крейна, развитыми в работах [20]-[23]. В настоящей работе мы используем результаты статьи [20], где эти методы получили дальнейшее развитие.

Мы используем систему единиц, в которой $c=\hbar=1$.

\section{2. САМОСОПРЯЖЕННЫЙ ГАМИЛЬТОНИАН В КУЛОНОВСКИХ И АБ-ПОТЕНЦИАЛАХ В $2+1$ ИЗМЕРЕНИЯХ}

В случае двух пространственных измерений пространство квантовых состояний частиц есть гильбертово пространство $\mathfrak{H}=L^{2}\left(\mathbb{R}^{2}\right)$ квадратично-интегрируемых функций $\Psi(\mathbf{r}), \mathbf{r}=(x, y)$ со скалярным произведением

$$
\left(\Psi_{1}, \Psi_{2}\right)=\int \Psi_{1}^{\dagger}(\mathbf{r}) \Psi_{2}(\mathbf{r}) d \mathbf{r}, \quad d \mathbf{r}=d x d y .
$$

Уравнение Дирака в $2+1$ измерениях для фермиона массы $m$ и заряда $e=-e_{0}<0$ в векторном и скалярном потенциалах $A_{\mu}$ и $U$ имеет вид

$$
\left(\gamma^{\mu} P_{\mu}-m-U\right) \Psi=0,
$$

где $P_{\mu}=-i \partial_{\mu}-e A_{\mu}$ - оператор обобщенного момента фермиона, а матрицы Дирака $\gamma^{\mu}$ удобно определить через двумерные матрицы Паули как

$$
\gamma^{0}=\sigma_{3}, \quad \gamma^{1}=i s \sigma_{1}, \quad \gamma^{2}=i \sigma_{2} .
$$

Здесь параметр $s$ характеризует два значения проекции спина фермиона $s= \pm 1$ [24].

Гамильтониан уравнения Дирака с параметром $s$ для фермиона в конфигурации потенциала АБ

$$
A_{0}=0, \quad A_{r}=0, \quad A_{\varphi}=\frac{B}{r},
$$


где $r=\sqrt{x^{2}+y^{2}}, \varphi=\operatorname{arctg}(y / x)$, в векторном и скалярном потенциалах $A_{\mu}$ и $U$, заданных формулами

$$
A_{0}(r)=\frac{a}{e_{0} r}, \quad A_{r}=0, \quad A_{\varphi}=0 ; \quad U(r)=-\frac{b}{r}, \quad a, b>0,
$$

есть

$$
H_{\mathrm{D}}=\sigma_{1} P_{2}-s \sigma_{2} P_{1}+\sigma_{3}(m+U(r))-e_{0} A_{0}(r) .
$$

Здесь векторный потенциал $A_{\mu}$ в операторе обобщенного импульса $P_{\mu}$ есть сумма выражений (4) и (5). Напомним, что $B$ характеризует магнитный поток: $\Phi=2 \pi B$, причем если $e B$ - целое число $N$, то полный магнитный поток $\Phi=2 \pi B$ квантован и $\Phi=\Phi_{0} N$, где $\Phi_{0} \equiv 2 \pi / e_{0}$.

Оператор Гамильтона (6) нужно определить как самосопряженный оператор в гильбертовом пространстве квадратично-интегрируемых спиноров $\Psi(\mathbf{r}), \mathbf{r}=(x, y)$ со скалярным произведением (1). Собственные значения оператора полного углового момента $J \equiv L_{z}+s \sigma_{3} / 2$, где $L_{z} \equiv-i \partial / \partial \varphi$ являются сохраняющимися величинами (оператор $J$ коммутирует с $H_{\mathrm{D}}$ ). Следовательно, можно рассматривать уравнение (2) отдельно в каждом собственном подпространстве $J$, а полное гильбертово пространство представить как прямую ортогональную сумму подпространств $J$.

Решения уравнения (2) в потенциалах (4) и (5) ищем в виде (см. работы [18], [25], [26])

$$
\Psi(t, \mathbf{r})=\frac{1}{\sqrt{2 \pi}} e^{-i E t+i l \varphi} \psi(r, \varphi),
$$

где вещественная величина $E$ - энергия фермиона, $l$ - целое число, а $\psi(r, \varphi)$ - двухкомпонентная функция (т. е. спинор)

$$
\psi(r, \varphi)=r^{-1 / 2}\left(\begin{array}{c}
f_{1}(r) \\
f_{2}(r) e^{i s \varphi}
\end{array}\right) .
$$

Волновая функция $\Psi$ является собственной функцией оператора полного углового момента $J$ с собственным значением $j=l+s / 2$.

Представляя спинор в виде (7), мы сводим уравнение (2) к радиальному уравнению Дирака в потенциалах (4) и (5) для функций $f_{1}(r)$ и $f_{2}(r)$ с формальным радиальным гамильтонианом $\check{h}$ :

$$
\check{h}=i s \sigma_{2} \frac{d}{d r}+\sigma_{1} \frac{l+\mu+s / 2}{r}-\frac{a}{r}+\left(m-\frac{b}{r}\right) \sigma_{3},
$$

где $\mu \equiv e_{0} B$. Тогда дублет

$$
F=\left(\begin{array}{l}
f_{1}(r) \\
f_{2}(r)
\end{array}\right)
$$

удовлетворяет уравнению

$$
\begin{aligned}
\check{h}\left(\begin{array}{l}
f_{1}(r) \\
f_{2}(r)
\end{array}\right) & =\left[\begin{array}{cc}
m-(a+b) / r & s d / d r+(l+\mu+s / 2) / r \\
-s d / d r+(l+\mu+s / 2) / r & -m-(a-b) / r
\end{array}\right]\left(\begin{array}{l}
f_{1}(r) \\
f_{2}(r)
\end{array}\right)= \\
& =E\left(\begin{array}{l}
f_{1}(r) \\
f_{2}(r)
\end{array}\right) .
\end{aligned}
$$


Нам понадобятся решения этого уравнения с комплексным параметром $E$, вещественные значения которого имеют смысл энергии. Таким образом, задача сводится к задаче для формального радиального гамильтониана $\check{h}$ для каждого $l=$ $0, \pm 1, \pm 2 \ldots$ и $s$ и произвольных $a, b, B$. При ее решении мы следуем работе [20].

Мы будем различать так называемые дифференциальные выражения $\check{k}$ и операторы $k$ и будем называть $k$ оператором, ассоциированным с дифференциальным выражением $\check{k}$. Пусть $\mathfrak{H}=\mathfrak{L}^{2}(0, \infty)$ есть гильбертово пространство дублетов $F(r)$, $G(r)$ со скалярным произведением

$$
(F, G)=\int_{0}^{\infty} F^{\dagger}(r) G(r) d r=\int_{0}^{\infty}\left[\bar{f}_{1}(r) g_{1}(r)+\bar{f}_{2}(r) g_{2}(r)\right] d r
$$

так что $\mathfrak{L}^{2}(0, \infty)=L^{2}(0, \infty) \oplus L^{2}(0, \infty)$. Здесь символом $\oplus$ обозначена прямая сумма. Определим оператор $h^{0}$ в гильбертовом пространстве $\mathfrak{L}^{2}(0, \infty)$ :

$$
h^{0}:\left\{\begin{array}{l}
D\left(h^{0}\right)=\mathcal{D}(0, \infty), \\
h^{0} F(r)=\check{h} F(r),
\end{array}\right.
$$

где $\mathcal{D}(0, \infty)=D(0, \infty) \oplus D(0, \infty), D(0, \infty)$ - стандартное пространство гладких функций на $(0, \infty)$ с компактным носителем

$$
D(0, \infty)=f(r): f(r) \in \mathbb{C}^{\infty}, \quad \operatorname{supp} f \in[c, d], \quad 0<c<d<\infty,
$$

отрезок $[c, d]$ может быть разным для разных $f$. Оператор $h$ является симметрическим, если для любых дублетов $F(r)$ и $G(r)$

$$
\int_{0}^{\infty} G^{\dagger}(r) h F(r) r d r=\int_{0}^{\infty}[h G(r)]^{\dagger} F(r) r d r .
$$

Очевидно, что $h^{0}$ - симметрический оператор.

Пусть $h$ есть самосопряженное расширение $h^{0}$ в $\mathfrak{L}^{2}(0, \infty)$. Область определения самосопряженного дифференциального оператора можно задать так называемыми самосопряженными граничными условиями на концах интервала для функций из области определения. В нашем случае, когда коэффициентная функция перед производной $d / d r$ не зависит от $r$, а свободные члены ограниченны при $r \rightarrow \infty$, проблема самосопряженных граничных условий связана только с левым концом $r=0$.

Рассмотрим оператор $h^{*}$, сопряженный исходному симметрическому оператору $h^{0}$. Нетрудно показать, что сопряженный оператор $h^{*}$, который формально определяется формулой (11), задается следующим образом:

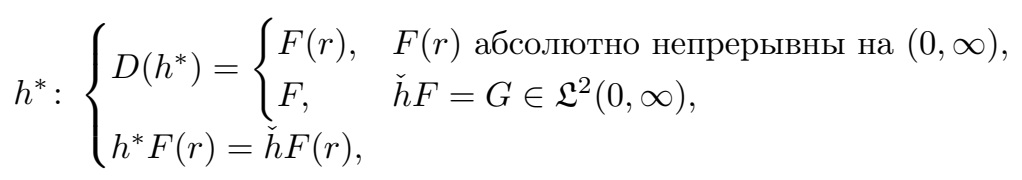

т. е. $h^{*}$ имеет более широкую область определения $D\left(h^{*}\right), D\left(h^{0}\right) \subset D\left(h^{*}\right)$, которая представляет собой так называемую естественную область для дифференциального выражения $\check{h}$. Вещественность коэффициентных функций дифференциального выражения (9) означает, что индексы дефекта исходного симметрического оператора $h^{0}$ равны, так что самосопряженные расширения оператора $h^{0}$ существуют для 
любых значений параметров $a, b, \mu$ и для каждого $l$ и $s$. Напомним, что симметрический оператор $k$ является самосопряженным, если $D(k)$ совпадает с областью определения сопряженного оператора $D\left(k^{*}\right)$.

Интегрируя по частям выражение (12), представим условие симметричности оператора в виде

$$
\lim _{r \rightarrow 0} G^{\dagger}(r) i \sigma_{2} F(r)=\lim _{r \rightarrow \infty} G^{\dagger}(r) i \sigma_{2} F(r) .
$$

Можно проверить, что для любого дублета $F(r)$ из $D\left(h^{*}\right)$

$$
\lim _{r \rightarrow \infty} F(r)=0,
$$

поэтому формула, которая фактически характеризует меру асимметрии сопряженного оператора $h^{*}$, сводится к граничным условиям в нуле:

$$
\lim _{r \rightarrow 0} G^{\dagger}(r) i \sigma_{2} F(r)=0 .
$$

Если условие (14) удовлетворяется для любых дублетов из $D\left(h^{*}\right)$, то $h^{*}$ является симметрическим и поэтому самосопряженным оператором. Это также означает, что $h^{0}$ - существенно самосопряженный оператор, и его единственным самосопряженным расширением является его замыкание $h=\bar{h}^{0}$, которое совпадает с сопряженным оператором: $h=h^{*}=h^{\dagger}$. Если условие (14) не удовлетворяется, самосопряженный оператор $h=h^{\dagger}$ находится как сужение оператора $h^{*}$ на так называемую максимальную область $D(h) \subset D\left(h^{*}\right)$ [27].

\section{3. РЕШЕНИЯ РАДИАЛЬНОГО УРАВНЕНИЯ ДИРАКА}

Функции $f_{1}(r)$ и $f_{2}(r)$ системы

$$
\begin{aligned}
& s \frac{d f_{1}}{d r}-\frac{l+\mu+s / 2}{r} f_{1}+\left(E+m+\frac{a-b}{r}\right) f_{2}=0, \\
& s \frac{d f_{2}}{d r}+\frac{l+\mu+s / 2}{r} f_{2}-\left(E-m+\frac{a+b}{r}\right) f_{1}=0
\end{aligned}
$$

ищем в виде (см. работы [19], [28])

$$
\begin{aligned}
& f_{1}(r)=\sqrt{m+E} e^{-x / 2} x^{\gamma_{s}}\left(Q_{1}^{s}+Q_{2}^{s}\right), \\
& f_{2}(r)=\sqrt{m-E} e^{-x / 2} x^{\gamma_{s}}\left(Q_{1}^{s}-Q_{2}^{s}\right),
\end{aligned}
$$

где

$$
x=2 \lambda r, \quad \lambda=\sqrt{m^{2}-E^{2}},
$$

a

$$
\gamma_{s}= \pm \sqrt{\left(l+\mu+\frac{s}{2}\right)^{2}-a^{2}+b^{2}} \equiv \gamma_{s}^{ \pm}
$$

определяет асимптотическое поведение решений при малых $r$.

Нетрудно показать, что функции $Q_{1}^{s}, Q_{2}^{s}$ удовлетворяют уравнениям

$$
x \frac{d^{2} Q_{1,2}^{s}}{d x^{2}}+\left[c_{s}-x\right] \frac{d Q_{1,2}^{s}}{d x}-a_{1,2}^{s} Q_{1,2}^{s}=0
$$


при

$$
a_{1,2}^{s}=\gamma_{s}+\frac{1}{2} \mp \frac{s}{2}-\frac{a E+m b}{\lambda}, \quad c_{s}=2 \gamma_{s}+1, \quad s= \pm 1,
$$

причем $a_{1}^{s}\left(a_{2}^{s}\right)$ определяется формулой (20) с верхним (нижним) знаком.

Решениями уравнения (19) являются вырожденные гипергеометрические функции $\Phi(a, c ; x)(\Phi(a, c ; x)$ не определена при $c=0,-1,-2, \ldots)$ и $\Psi(a, c ; x)[29] ;$ общее решение можно представить в виде

$$
Q_{1,2}^{s}=A_{1,2} \Phi\left(a_{1,2}^{s}, c_{s} ; x\right)+B_{1,2} \Psi\left(a_{1,2}^{s}, c_{s} ; x\right),
$$

где $A$ и $B$ - константы; связи между $A_{1}, B_{1}$ и $A_{2}, B_{2}$ можно найти, положив в каком-либо из уравнений (15) $r=0$ :

$$
\begin{gathered}
A_{2}=\frac{s \gamma_{s}^{ \pm}-(E a+m b) / \lambda}{\nu+(m a+b E) / \lambda} A_{1}, \\
B_{2}=\frac{\left[(E a+m b)^{2} / \lambda^{2}-\left(\gamma_{s}\right)^{2}-1\right](s+1) / 2+1}{\nu+(a m+b E) / \lambda} B_{1}, \quad \nu=l+\mu+\frac{s}{2} .
\end{gathered}
$$

Нелишне напомнить соотношение между функциями $\Psi$ и $\Phi$ :

$$
\Psi(a, c ; x)=\frac{\Gamma(1-c)}{\Gamma(a-c+1)} \Phi(a, c ; x)+\frac{\Gamma(c-1)}{\Gamma(a)} x^{1-c} \Phi(a-c+1,2-c ; x),
$$

где $\Gamma(z)$ - гамма-функция Эйлера.

Запишем дублет $F$ как

$$
\begin{gathered}
F=\left(\begin{array}{c}
f_{1} \\
f_{2}
\end{array}\right)=e^{-x / 2} x^{\gamma_{s}} A\left[v_{+} \Phi\left(a_{1}^{s}, c_{s} ; x\right)+v_{-} m_{s}^{+} \Phi\left(a_{1}^{s}+s, c_{s} ; x\right)\right]+ \\
+e^{-x / 2} x^{\gamma_{s}} B\left[v_{+} \Psi\left(a_{1}^{s}, c_{s} ; x\right)+v_{-} n_{s}^{+} \Psi\left(a_{1}^{s}+s, c_{s} ; x\right)\right] .
\end{gathered}
$$

Здесь мы обозначили

$$
\begin{gathered}
m_{s}^{ \pm}=\frac{\left(s \gamma_{s}^{ \pm}\right)-(E a+m b) / \lambda}{\nu+(a m+b E) / \lambda}, \quad n_{s}^{ \pm}=\frac{\left[(E a+m b)^{2} / \lambda^{2}-\gamma_{s}^{2}-1\right](s+1) / 2+1}{\nu+(a m+b E) / \lambda}, \\
v_{+}=\left(\begin{array}{c}
1 \\
p
\end{array}\right), \quad v_{-}=\left(\begin{array}{c}
1 \\
-p
\end{array}\right), \quad p=\sqrt{\frac{m-E}{m+E}} .
\end{gathered}
$$

Тогда общее решение радиальных уравнений удобно представить в виде

$$
F=\left(\begin{array}{c}
f_{1} \\
f_{2}
\end{array}\right)=A Y\left(r, \gamma_{s}, E\right)+B e^{-x / 2} x^{\gamma_{s}}\left[\Psi\left(a_{s}^{1}, c_{s} ; x\right) v_{+}+n_{s}^{+} \Psi\left(a_{s}^{1}+s, c_{s} ; x\right) v_{-}\right]
$$

Здесь

$$
A\left(1-m_{s}^{+}\right) p\left(\frac{2 \lambda}{m}\right)^{\gamma_{s}} \rightarrow A
$$

а дублет

$$
Y\left(r, \gamma_{s}, E\right)=\frac{(m r)^{\gamma_{s}}}{2}\left[\Phi_{+}\left(r, \gamma_{s}, E\right)+\frac{s}{\lambda}\left(\begin{array}{cc}
0 & m+E \\
m-E & 0
\end{array}\right) \Phi_{-}\left(r, \gamma_{s}, E\right)\right] u_{+},
$$


где

$$
\begin{gathered}
\Phi_{+}=e^{-x / 2} \Phi\left(a_{1}^{s}, c_{s} ; x\right)+e^{x / 2} \Phi\left(c_{s}-a_{2}^{s}-1, c_{s} ;-x\right), \\
\Phi_{-}=e^{-x / 2} \Phi\left(a_{1}^{s}, c_{s} ; x\right)-e^{x / 2} \Phi\left(c_{s}-a_{2}^{s}-1, c_{s} ;-x\right), \\
u_{ \pm}=\left(\begin{array}{c}
\left(s \gamma_{s}^{ \pm}+\nu\right) /(a+b) \\
1
\end{array}\right) .
\end{gathered}
$$

Приведем некоторые полезные частные решения системы (15). Одно из них дается формулой (25) с $A=1, B=0$ при специальном выборе $\gamma_{s}$ :

$$
U_{1}(r ; E)=\left.Y\left(r, \gamma_{s}, E\right)\right|_{\gamma_{s}=\gamma_{s}^{+}},
$$

где

$$
\gamma_{s}^{+}= \begin{cases}\sqrt{\nu^{2}-\left(a^{2}-b^{2}\right)}, & \sqrt{a^{2}-b^{2}} \leqslant|\nu|, \\ i \sqrt{\left(a^{2}-b^{2}\right)-\nu^{2}}, & \sqrt{a^{2}-b^{2}}>|\nu| .\end{cases}
$$

Обозначим $\gamma=\sqrt{\nu^{2}-\left(a^{2}-b^{2}\right)}, \sigma=\sqrt{\left(a^{2}-b^{2}\right)-\nu^{2}}$. Асимптотическое поведение дублета $U_{1}(r ; E)$ при $r \rightarrow 0$ имеет вид

$$
U_{1}(r ; E)=(m r)^{\gamma_{s}^{+}} u_{+}+O\left(r^{\gamma_{s}^{+}+1}\right) .
$$

При $\gamma_{s}^{+} \neq n / 2, n=1,2, \ldots$, решение

$$
U_{2}(r ; E)=\left.Y\left(r, \gamma_{s}, E\right)\right|_{\gamma_{s}=-\gamma_{s}^{+}}
$$

с асимптотическим поведением при $r \rightarrow 0$

$$
U_{2}(r ; E)=(m r)^{-\gamma_{s}^{+}} u_{-}+O\left(r^{-\gamma_{s}^{+}+1}\right)
$$

также будет использовано. Решения $U_{1}(r ; E)$ и $U_{2}(r ; E)$ линейно независимы, так каK

$$
\mathrm{Wr}\left(U_{1}, U_{2}\right)=\frac{2 s \gamma_{s}^{+}}{a+b}
$$

Здесь $\operatorname{Wr}\left(U_{1}, U_{2}\right)$ - вронскиан, который для дублетов $F=\left(\begin{array}{l}f_{1} \\ f_{2}\end{array}\right)$ и $G=\left(\begin{array}{l}g_{1} \\ g_{2}\end{array}\right)$ определяется формулой

$$
\mathrm{Wr}(F, G)=F i \sigma^{2} G=f_{1} g_{2}-f_{2} g_{1} .
$$

Другое нужное решение, нетривиальное при $\gamma_{s}^{+} \neq n / 2, n=1,2, \ldots$, дается формулой (25) с $A=0$ при специальном выборе $B$ :

$$
\begin{gathered}
V_{1}(r ; E)=B(E)(m r)^{\gamma_{s}^{+}} e^{-x / 2}\left[\Psi\left(a_{1}^{s}, c_{s}, x\right) v^{+}+n_{s}^{+} \Psi\left(a_{1}^{s}+s, c_{s}, x\right) v^{-}\right], \\
B(E)=\frac{\Gamma\left(-\gamma_{s}^{+}+(1-s) / 2-(a E+m b) / \lambda\right)}{\Gamma\left(-2 \gamma_{s}^{+}\right)\left(1-m_{s}^{+}\right)} .
\end{gathered}
$$

Решение $V_{1}$ можно записать в виде линейной комбинации $U_{1}$ и $U_{2}$ :

$$
V_{1}(r ; E)=U_{1}(r ; E)+\frac{a+b}{2 s \gamma_{s}^{+}} \omega(E) U_{2}(r ; E),
$$


где

$$
\begin{aligned}
\omega(E) & \equiv \operatorname{Wr}\left(U_{1}, V_{1}\right)= \\
& =\frac{\Gamma\left(2 \gamma_{s}^{+}\right) \Gamma\left(-\gamma_{s}^{+}+(1-s) / 2-(a E+m b) / \lambda\right)}{\Gamma\left(-2 \gamma_{s}^{+}\right) \Gamma\left(\gamma_{s}^{+}+(1-s) / 2-(a E+m b) / \lambda\right)} \frac{(2 \lambda)^{-2 \gamma_{s}^{+}}}{m^{-2 \gamma_{s}^{+}}} \frac{\left(1-m_{s}^{-}\right)}{\left(1-m_{s}^{+}\right)} \frac{2 s \gamma_{s}^{+}}{a+b} \equiv \\
& \equiv \frac{\widetilde{\omega}(E)}{\Gamma\left(-2 \gamma_{s}^{+}\right)} .
\end{aligned}
$$

Дублеты $U_{2}$ и $V_{1}$ не являются линейно независимыми решениями при $\gamma_{s}^{+}=n / 2$, $n=1,2, \ldots$; для этих значений дублет $U_{2}$ не определен, а $V_{1}$ равен нулю. Однако для этих частных значений $\gamma_{s}^{+}$можно построить аналоги дублетов $U_{2}$ и $V_{1}$. Такие дублеты, сконструированные вблизи некоторого фиксированного $n$, должны быть решениями радиальных уравнений, хорошо определенными как в некоторой окрестности точки $\gamma_{s}^{+}=n / 2$, так и в самой этой точке (мы следовали работе [20]).

Дублет $U_{2}$ имеет сингулярность вида $\Gamma\left(-2 \gamma_{s}^{+}\right)$при $\gamma_{s}^{+}=n / 2$ и в окрестности этой точки может быть представлен как

$$
U_{2}(r ; E)=\Gamma\left(-2 \gamma_{s}^{+}\right) A_{n}(E) U_{1}(r ; E)+U_{n(2)}(r ; E),
$$

где

$$
A_{n}(E)=\left.\left(\frac{2 s \gamma_{s}^{+}}{(a+b) \widetilde{\omega}(E)}\right)\right|_{\gamma_{s}^{+}=n / 2},
$$

a $U_{n(2)}(r ; E)$ имеет конечный предел при $\gamma_{s}^{+} \rightarrow n / 2$ и удовлетворяет радиальным уравнениям (15). Дублет

$$
U_{n(2)}(r ; E)=U_{2}(r ; E)-\Gamma\left(-2 \gamma_{s}^{+}\right) A_{n}(E) U_{1}(r ; E)
$$

удовлетворяет условию

$$
U_{n(2)}(r ; E)=(m r)^{-\gamma_{s}^{+}} u_{-}+O\left(r^{-\gamma_{s}^{+}+1}\right), \quad r \rightarrow 0,
$$

и линейно независим от $U_{1}$, так как

$$
\mathrm{Wr}\left(U_{1}, U_{n(2)}\right)=\frac{2 s \gamma_{s}^{+}}{a+b} .
$$

Дублет $V_{1}$ согласно формулами (34), (35) можно представить в окрестности точки $\gamma_{s}^{+} \rightarrow n / 2$ в виде комбинации конечных дублетов $U_{1}$ и $U_{n(2)}$ :

$$
V_{1}(r ; E)=\left[1+\frac{a+b}{2 s \gamma_{s}^{+}} \widetilde{\omega}(E) A_{n}(E)\right] U_{1}(r ; E)+\frac{a+b}{2 s \gamma_{s}^{+}} \omega(E) U_{n(2)}(r ; E),
$$

где оба множителя $1+(a+b) \widetilde{\omega}(E) A_{n}(E) / 2 s \gamma_{s}^{+}$и $\omega(E)$ стремятся к нулю как $1 / \Gamma\left(-2 \gamma_{s}^{+}\right)$при $\gamma_{s}^{+} \rightarrow n / 2$. Поэтому дублет

$$
V_{n(1)}(r ; E)=\frac{1}{1+(a+b) \widetilde{\omega}(E) A_{n}(E) / 2 s \gamma_{s}^{+}} V_{1}(r ; E)=U_{1}(r ; E)+\frac{a+b}{2 s \gamma_{s}^{+}} \omega_{n}(E) U_{n(2)}(r ; E)
$$

при

$$
\omega_{n}(E)=\frac{\omega(E)}{1+(a+b) \widetilde{\omega}(E) A_{n}(E) / 2 s \gamma_{s}^{+}}=\frac{\widetilde{\omega}(E)}{\Gamma\left(-2 \gamma_{s}^{+}\right)\left[1+(a+b) \widetilde{\omega}(E) A_{n}(E) / 2 s \gamma_{s}^{+}\right]}
$$


является решением радиальных уравнений, хорошо определенным в некоторой окрестности точки $\gamma_{s}^{+}=n / 2$ и в самой этой точке. Функция $\omega_{n}(E)$ также хорошо определена в некоторой окрестности точки $\gamma_{s}^{+}=n / 2$ и в самой этой точке.

В частном (специальном) случае $\gamma_{s}^{+} \equiv \gamma=0$ дублеты $U_{1}$ и $U_{2}$ совпадают, а $V_{1}$ равен нулю. Тогда, если через $U_{1}(r ; E \mid \gamma), U_{2}(r ; E \mid \gamma)$ и $V_{1}(r ; E \mid \gamma)$ обозначить дублеты $U_{1}, U_{2}$ и $V_{1}$ при $\gamma \neq 0$, в качестве двух линейно независимых решений уравнений (15) можно выбрать (следуя работе [20])

$$
\begin{gathered}
U_{1}(r ; E)=U_{1}(r ; E \mid 0), \quad U_{1}(r ; E)=u_{+}+O(r), \quad r \rightarrow 0, \\
U_{2}^{0}(r ; E)=\left.\frac{\partial U_{1}(r ; E \mid \gamma)}{\partial \gamma}\right|_{\gamma=0}-\frac{s}{\nu} U_{1}(r ; E \mid 0), \\
U_{2}^{0}(r ; E)=u_{-}^{0}(r)+O(r \ln r), \quad r \rightarrow 0,
\end{gathered}
$$

где

$$
u_{+}=\left(\begin{array}{c}
\nu /(a+b) \\
1
\end{array}\right), \quad u_{-}^{0}(r)=\left(\begin{array}{c}
\nu \ln (m r) /(a+b) \\
\ln (m r)-s / \nu
\end{array}\right) .
$$

Вронскиан этих решений имеет вид

$$
\operatorname{Wr}\left(U_{1}, U_{2}^{0}\right)=-\frac{s}{a+b} .
$$

Аналогом дублета $V_{1}$ при $\gamma=0$ является

$$
\begin{aligned}
V_{1}^{0}(r ; E) & =\lim _{\gamma \rightarrow 0}\left[-\Gamma(-2 \gamma) V_{1}(r ; E \mid \gamma)\right]= \\
& =-\frac{\Gamma\left(a_{s}\right)}{\left(1-m_{s}\right)} e^{-x / 2}\left[\Psi\left(a_{s}, 1, x\right) v^{+}+n_{s} \Psi\left(a_{s}+s, 1, x\right) v^{-}\right]
\end{aligned}
$$

при

$$
\begin{gathered}
a_{s}=\frac{1-s}{2}-\frac{a E+m b}{\lambda}, \quad x=2 \lambda r \\
m_{s}=\frac{-(E a+m b) / \lambda}{\nu+(a m+b E) / \lambda}, \quad n_{s}=\frac{\left[(E a+m b)^{2} / \lambda^{2}-1\right]((s+1) / 2)+1}{\nu+(a m+b E) / \lambda} .
\end{gathered}
$$

Дублет $V_{1}^{0}$ можно представить как комбинацию $U_{1}$ и $U_{2}^{0}$ :

$$
V_{1}^{0}(r ; E)=U_{2}^{0}(r ; E)+\frac{a+b}{s} \omega^{(0)}(E) U_{1}(r ; E),
$$

где

$$
\begin{aligned}
\omega^{(0)}(E)= & \operatorname{Wr}\left(U_{2}^{0}(r ; E), V_{1}^{0}(r ; E)\right)= \\
= & \frac{s}{a+b}\left[\ln \left(\frac{2 \lambda}{m}\right)+\psi\left(-\frac{1-s}{2}-\frac{a E+m b}{\lambda}\right)-\right. \\
& \left.-2 \psi(1)+\frac{s(a+b)(m+E)}{\nu^{2} \lambda+\nu(a+b)(m+E)}\right] .
\end{aligned}
$$

Здесь через $\psi(z)$ обозначена логарифмическая производная гамма-функции. 


\section{4. СПЕКТРЫ САМОСОПРЯЖЕННОГО РАДИАЛЬНОГО ГАМИЛЬТОНИАНА}

Задача нахождения спектров самосопряженных расширений радиального гамильтониана в кулоновском поле была сформулирована и решена в работе [20] на основе метода направляющих функционалов Крейна, в котором необходимо сконструировать направляющий функционал, построить функцию Грина и вычислить спектральную функцию. Здесь мы должны найти спектры самосопряженных расширений радиального гамильтониана в кулоновских и АБ-потенциалах в $2+1$ измерениях в гильбертовом пространстве $\mathfrak{L}^{2}(0, \infty)$ в различных областях значений параметров.

В дальнейшем будем обозначать область определения сопряженного оператора $h^{*}$ как $D^{*}$. Область определения самосопряженного оператора $h=h^{\dagger}$ находится как сужение оператора $h^{*}$ на максимальную область $D(h)$, поэтому любой дублет из этой области должен удовлетворять граничному условию (14), которое запишем как

$$
\left.\left(F^{\dagger}(r) i \sigma_{2} F(r)\right)\right|_{r=0}=\left.\left(\bar{f}_{1} f_{2}-\bar{f}_{2} f_{1}\right)\right|_{r=0}=0 .
$$

Мы называем граничные условия в форме (47) самосопряженными граничными условиями. Чтобы проверить выполнение (47), нам нужно знать асимптотическое поведение дублета $F$ при $r \rightarrow 0$.

Согласно определению (13) любые дублеты из $D^{*}$ можно рассматривать как квадратично-интегрируемые решения неоднородного дифференциального уравнения

$$
\check{h} F(r)=\left[i s \sigma_{2} \frac{d}{d r}+\sigma_{1} \frac{l+\mu+s / 2}{r}-\frac{a}{r}+\left(m-\frac{b}{r}\right) \sigma_{3}\right] F(r)=G(r),
$$

в котором дублет $G(r)$ принадлежит $\mathfrak{L}^{2}(0, \infty)$ и, следовательно, локально интегрируем. Таким образом, к уравнению (48) можно применить общую теорию дифференциальных уравнений [22].

Чтобы оценить асимптотическое поведение $F(r)$ при $r=0$, перепишем уравнение (48) в виде [20]

$$
\check{h}_{-} F(r)=G_{-}(r),
$$

где

$$
\check{h}_{-}=i s \sigma_{2} \frac{d}{d r}+\sigma_{1} \frac{l+\mu+s / 2}{r}-\frac{a}{r}-\frac{b}{r} \sigma_{3}, \quad G_{-}(r)=G(r)-m \sigma_{3} F(r)
$$

при $G_{-}(r)$, принадлежащей $\mathfrak{L}^{2}(0, \infty)$.

Запишем выражение (18) в форме $q=\sqrt{\nu^{2}-\left(\gamma_{s}^{+}\right)^{2}}$ и введем

$$
q_{u}=\sqrt{\nu^{2}-\frac{1}{4}} \quad \Leftrightarrow \quad \gamma_{s}^{+}=\frac{1}{2}, \quad q_{\mathrm{c}}=\nu \quad \Leftrightarrow \quad \gamma_{s}^{+}=0 .
$$

Величина $q$ играет роль некоторого эффективного заряда, являющегося функцией $l, s, a, b, \mu$. Здесь мы рассмотрим случай $a>b$.

Пусть $U_{1}(r)$ и $U_{2}(r)$ - линейно независимые решения однородного дифференциального уравнения $h_{-} F(r)=0$ :

$$
\begin{aligned}
& U_{1}(r)=(m r)^{\gamma_{s}^{+}} u_{+}, \quad q>0, \\
& U_{2}(r)= \begin{cases}(m r)^{-\gamma_{s}^{+}} u_{-}, & q>0, \quad q \neq q_{\mathrm{c}} \quad\left(\gamma_{s}^{+} \neq 0\right), \\
u_{-}^{0}(r), & q=q_{\mathrm{c}} \quad\left(\gamma_{s}^{+}=0\right) .\end{cases}
\end{aligned}
$$


Любое решение неоднородного дифференциального уравнения можно представить в форме [20]

$$
F(r)=c_{1} U_{1}(r)+c_{2} U_{2}(r)+I_{1}(r)+I_{2}(r),
$$

где $c_{1}$ и $c_{2}-$ некоторые комплексные константы и

$$
\begin{aligned}
& I_{1}(r)= \begin{cases}-\frac{a+b}{2 s \gamma_{s}^{+}} \int_{r}^{r_{0}}\left[U_{1}(r) \otimes U_{2}(y)\right] G_{-}(y) d y, & 0<q \leqslant q_{u}, \\
\frac{a+b}{2 s \gamma_{s}^{+}} \int_{0}^{r}\left[U_{1}(r) \otimes U_{2}(y)\right] G_{-}(y) d y, & q>q_{u}, \quad q \neq q_{\mathrm{c}} \\
-\frac{a+b}{s} \int_{0}^{r}\left[U_{1}(r) \otimes U_{2}(y)\right] G_{-}(y) d y, & q=q_{\mathrm{c}},\end{cases} \\
& I_{2}(r)= \begin{cases}-\frac{a+b}{2 s \gamma_{s}^{+}} \int_{0}^{r}\left[U_{2}(r) \otimes U_{1}(y)\right] G_{-}(y) d y, & q>0, \quad q \neq q_{\mathrm{c}} \\
\frac{a+b}{s} \int_{0}^{r}\left[U_{2}(r) \otimes U_{1}(y)\right] G_{-}(y) d y, & q=q_{\mathrm{c}} .\end{cases}
\end{aligned}
$$

Здесь $G_{-}(y)$ - правая часть уравнения (49), символом $\otimes$ обозначено тензорное произведение, так что $\left[U_{1}(r) \otimes U_{2}(y)\right]$ есть $(2 \times 2)$-матрица, а $r_{0}$ - вещественная постоянная. Можно показать, что асимптотическое поведение дублета $F(r)$ при $r \rightarrow 0$ определяется двумя первыми членами в формуле (52) и, следовательно, существенно зависит от значения $\gamma_{s}^{+}[20]$.

4.1. Малые эффективные заряды $q \leqslant q_{u}$. При малых $q$ :

$$
0<q \leqslant q_{u} \Longleftrightarrow \gamma_{s}^{+}=\gamma \geqslant \frac{1}{2}
$$

только функция $U_{1}(r) \propto r^{\gamma_{s}^{+}}$квадратично-интегрируема в точке $r=0$, но не $U_{2}(r) \propto$ $r^{-\gamma_{s}^{+}}$. Интегралы $I_{1}(r)$ и $I_{2}(r)$ можно оценить, используя неравенство Коши-Буняковского, что дает

$$
I_{1}(r)=O\left(r^{1 / 2}\right), \quad I_{2}(r)=O\left(r^{1 / 2}\right), \quad r \rightarrow 0 .
$$

Для принадлежности дублета $F(r)$ гильбертову пространству $\mathfrak{L}^{2}(0, \infty)$ необходимо, чтобы выполнялось $c_{2}=0$ :

$$
F(r)=c_{1} U_{1}(r)+I_{1}(r)+I_{2}(r)=O\left(r^{1 / 2}\right) \rightarrow 0, \quad r \rightarrow 0 .
$$

Тогда $F \in D^{*}$ и удовлетворяет (47). Это означает, что в области (55) индексы дефекта исходного симметрического оператора $h^{0}$ равны нулю, т. е. оператор $h$ является существенно самосопряженным: $h=h^{\dagger}$, так что при $\gamma \geqslant 1 / 2$ существует единственный самосопряженный радиальный гамильтониан $h=h^{0}$. Область его определения $D(h)$ есть пространство абсолютно непрерывных дублетов $F(r)$, исчезающих в точке $r=0$; дублет $\check{h} F(r)$ также принадлежит $\mathfrak{L}^{2}(0, \infty)$.

Мы не будем приводить выражения для направляющего функционала и функции Грина, однако отметим, что направляющий функционал простой; это подразумевает, что и спектр оператора $h$ простой. Спектр самосопряженного оператора можно 
найти с помощью спектральной функции; выражение для ее производной дается формулой

$$
\frac{d \sigma(E)}{d E}=\frac{1}{\pi} \lim _{\epsilon \rightarrow 0} \operatorname{Im} \frac{1}{\omega(E+i \epsilon)},
$$

где $\lim$, как и $d \sigma(E) / d E$, понимается в обобщенном смысле [20], т. е. функция $\omega(E)=$ $\lim _{\epsilon \rightarrow 0} \omega(E+i \epsilon)$ рассматривается как обобщенная функция, которую можно получить аналитическим продолжением вронскиана (36) в комплексную плоскость $E$. На действительной оси $E$ обобщенная функция совпадает с соответствующей функцией действительной переменной. Таким образом, спектральная функция определяется обобщенной функцией $F(E)=\lim _{\epsilon \rightarrow 0} \omega^{-1}(E+i \epsilon)$. В точках, в которых функция $\omega(E)=\lim _{\epsilon \rightarrow 0} \omega(E+i \epsilon)$ отлична от нуля, $F(E)=1 / \omega(E)$.

В области $|E|<m$ выражение

$$
\omega(E)=\frac{\Gamma\left(2 \gamma_{s}^{+}\right) \Gamma\left(-\gamma_{s}^{+}+(1-s) / 2-(a E+m b) / \lambda\right)}{\Gamma\left(-2 \gamma_{s}^{+}\right) \Gamma\left(\gamma_{s}^{+}+(1-s) / 2-(a E+m b) / \lambda\right)} \frac{(2 \lambda)^{-2 \gamma_{s}^{+}}}{m^{-2 \gamma_{s}^{+}}} \frac{\left(1-m_{s}^{-}\right)}{\left(1-m_{s}^{+}\right)} \frac{2 s \gamma_{s}^{+}}{a+b}
$$

является вещественным, поэтому $\lim _{\epsilon \rightarrow 0}[1 / \omega(E+i \epsilon)]$ может принимать комплексные значения только в точках, в которых $\omega(E)=0$, т. е. когда или

$$
1-m_{s}^{-} \equiv \nu+\frac{(a+b)(m+E)}{\lambda}+s \gamma_{s}^{+}=0
$$

или

$$
\gamma_{s}^{+}+\frac{1-s}{2}-\frac{a E+m b}{\lambda}=-n, \quad n=0,1, \ldots ;
$$

в этих точках $\Gamma\left(\gamma_{s}^{+}+(1-s) / 2-(a E+m b) / \lambda\right)=\infty$. Можно показать, что в точках, определяемых выражением $(60), \omega(E) \neq 0$. Следовательно, дискретный спектр энергий связанных состояний фермионов определяется соотношением (61) и имеет вид

$$
\frac{E_{n, l}}{m}=\sqrt{\left(\frac{a b}{v^{2}+a^{2}}\right)^{2}+\frac{v^{2}-b^{2}}{v^{2}+a^{2}}}-\frac{a b}{v^{2}+a^{2}},
$$

где $v=n+\gamma_{s}^{+}+(1-s) / 2$. Для $s=1, n=0$ из формулы $(61)$ видно, что $(E a+m b) / \lambda=$ $\gamma>0$, откуда и из легко проверяемого равенства

$$
\left(\gamma_{s}^{+}\right)^{2}-\frac{a^{2}-b^{2}}{\lambda^{2}} E^{2}=\nu^{2}-\frac{a^{2}-b^{2}}{\lambda^{2}} m^{2}
$$

получим $(E b+m a) / \lambda=|\nu|$. Следовательно, $1-m_{s}^{+}=0$ для $\nu<0$. Поэтому произведение $\left|\Gamma(\gamma+(1-s) / 2-(a E+m b) / \lambda)\left(1-m_{s}^{+}\right)\right|<\infty$ для $s=1, n=0, \nu<0$, так что $\omega(E) \neq 0$. Таким образом, допустимы следующие значения $n: n=0,1,2, \ldots$, $s= \pm 1$ для $\nu>0$ и $n=1,2, \ldots, s=1, n=0,1,2, \ldots, s=-1$ для $\nu<0$. Заметим, что магнитный поток влияет на спектр энергий фермиона через величину $\mu$, которая входит в $\gamma_{s}^{+}$.

Все уровни энергии за исключением основного (низшего) уровня дважды вырожденны по $s$. Основное состояние фермиона в рассматриваемой конфигурации полей не вырожденно; это состояние с $n=0, s=1$ для $\nu>0$, что при заданном $e$ означает $\mu>0(B>0)$, т. е. $s \mu>0$. В основном состоянии, как в состоянии с наименьшей 
энергией, потенциальная энергия взаимодействия спинового магнитного момента фермиона с магнитным полем, задаваемая оператором $-s \mu \delta(r) / r$, должна быть минимальной, поэтому $s \mu>0$. Если представить магнитный поток в единицах $\Phi_{0}$ в форме

$$
\mu=[\mu]+\beta \equiv N+\beta,
$$

где $[\mu] \equiv N$ - целое число $(\leqslant \mu)$,

$$
1>\beta \geqslant 0,
$$

то $N=0,1,2, \ldots$ для $\mu>0$. Для $\mu>0$ основное состояние фермиона (т. е. частицы с зарядом $e$ ) есть состояние с $s=1$ и энергией

$$
\frac{E_{g}}{m}=\sqrt{\left(\frac{a b}{(\beta+1 / 2)^{2}+b^{2}}\right)^{2}+\frac{(\beta+1 / 2)^{2}-a^{2}}{(\beta+1 / 2)^{2}+b^{2}}}-\frac{a b}{(\beta+1 / 2)^{2}+b^{2}} .
$$

При фиксированном знаке $e$ переход к случаю $\nu<0, \mu<0(B<0)$ подразумевает одновременные замены $l \rightarrow-l, B \rightarrow-B, s \rightarrow-s$, при которых $s \mu>0$. Поэтому, для $\mu<0$ основное состояние фермиона (с зарядом $e$ ) есть состояние с $s=-1$ и с энергией (66). Этот результат понятен с точки зрения физики: при изменении знака напряженности магнитного поля энергия взаимодействия спинового магнитного момента фермиона с магнитным полем будет минимальной, если при этом изменить знак проекции спина фермиона на направление поля.

Нетрудно показать, что спектр сгущается в точке $E=m$, и его асимптотический вид при $n \rightarrow \infty$ дается нерелятивистской формулой

$$
\epsilon_{n}=m-E_{n}=\frac{m(a+b)^{2}}{2 n^{2}} .
$$

Интересно, что эта формула совпадает с формулой, описывающей нерелятивистский дискретный спектр энергий связанных состояний фермиона в чисто кулоновском векторном потенциале (5), в которую вместо $a$ входит сумма $a+b$.

4.2. Область эффективных зарядов $q_{u}<q<q_{\mathrm{c}}$. Эффективные заряды со

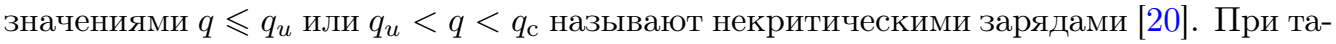
ких зарядах не возникает проблем с определением спектров энергий стандартными методами квантовой механики. Во второй некритической области

$$
q_{u}<q<q_{\mathrm{c}} \Longleftrightarrow 0<\gamma_{s}^{+}=\gamma<\frac{1}{2}
$$

обе функции $U_{1}(r) \propto r^{\gamma_{s}^{+}}$и $U_{2}(r) \propto r^{-\gamma_{s}^{+}}$квадратично-интегрируемы в точке $r=$ 0 . Оценки интегралов $I_{1}(r)$ и $I_{2}(r)$, сделанные выше, остаются справедливыми, поэтому любой дублет $F \in D^{*}$ ведет себя при $r \rightarrow 0$ как

$$
F(r)=c_{1}(m r)^{\gamma_{s}^{+}} u_{+}+c_{2}(m r)^{-\gamma_{s}^{+}} u_{-}+O\left(r^{1 / 2}\right), \quad u_{ \pm}=\left(\begin{array}{c}
\left(s \gamma_{s}^{ \pm}+\nu\right) /(a+b) \\
1
\end{array}\right)
$$

Однако дублет с таким поведением не удовлетворяет условию (47), так как

$$
\left.\left(\bar{f}_{1} f_{2}-\bar{f}_{2} f_{1}\right)\right|_{r=0}=\frac{2 s \gamma}{a+b}\left(\bar{c}_{1} c_{2}-\bar{c}_{2} c_{1}\right) .
$$


Это означает, что оператор $h^{*}$ не является симметрическим, и необходимо построить нетривиальные самосопряженные расширения оператора $h^{0}$. Эта задача для случая кулоновского поля решена в работе [20]. С помощью линейного преобразования

$$
c_{1,2} \rightarrow c_{ \pm}=c_{1} \pm i c_{2}
$$

соотношение (70) можно привести к квадратичной диагональной форме:

$$
\left.\left(\bar{f}_{1} f_{2}-\bar{f}_{2} f_{1}\right)\right|_{r=0}=-i \frac{s \gamma}{a+b}\left(\left|c_{+}\right|^{2}-\left|c_{-}\right|^{2}\right) .
$$

Индексы инерции этой формы равны $(1,1)$, поэтому индексы дефекта симметрического оператора $h^{0}$ для $0<\gamma<1 / 2$ равны $(1,1)$.

Условие (47) будет удовлетворяться для любых $c_{-}$и $c_{+}$, связанных соотношением

$$
c_{-}=e^{i \theta} c_{+}, \quad 0 \leqslant \theta \leqslant 2 \pi, \quad 0 \sim 2 \pi .
$$

Угол $\theta$ параметризует самосопряженные расширения $\left(h_{\theta}\right)$ исходного симметрического оператора $h^{0}$, которые различны для разных $\theta$ за исключением двух эквивалентных значений: $\theta=0$ и $\theta=2 \pi$.

Соотношение (73) можно записать в эквивалентной форме

$$
c_{2}=-\xi c_{1}, \quad-\infty \leqslant \xi=\operatorname{tg} \frac{\theta}{2} \leqslant+\infty, \quad-\infty \sim+\infty
$$

В терминах $\xi$ значения $\xi= \pm \infty$ эквивалентны и подразумевают, что $c_{1}=0$. Мы будем считать, что в этих случаях $\xi=\infty$.

Следовательно, в области $0<\gamma_{s}^{+}<1 / 2$ можно определить однопараметрическое $U(1)$-семейство самосопряженных операторов $h_{\theta} \equiv h_{\xi}$ с областью определения $D_{\xi}$ :

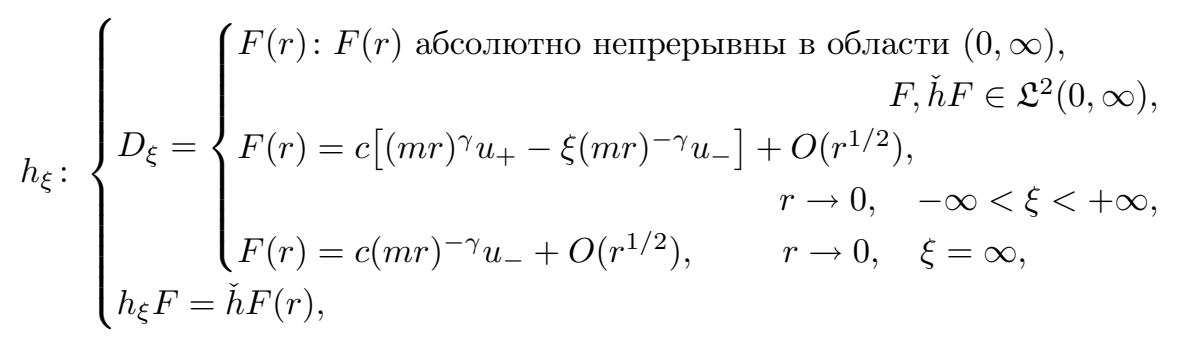

где $c$ - произвольная комплексная постоянная. Другими словами, в случае $0<\gamma_{s}^{+}<$ $1 / 2$ исходный симметрический оператор $h^{0}$ нельзя определить как единственный самосопряженный оператор, и поэтому область его определения нужно дополнительно фиксировать с помощью вещественного параметра $\xi$ в терминах самосопряженных граничных условий. Для $\xi=0$ весь анализ аналогичен проведенному в предыдущем пункте, и все полученные в нем формулы остаются справедливыми и в случае $0<\gamma_{s}^{+}<1 / 2$.

Далее рассмотрим случай $0<|\xi|<\infty$. В качестве дублета $U_{\xi}(r ; E)$, определяющего направляющий функционал, выберем

$$
U_{\xi}(r ; E)=U_{1}(r ; E)-\xi U_{2}(r ; E) \text {. }
$$


Нетрудно показать, что направляющий функционал в этом случае простой. При $r \rightarrow 0$

$$
U_{\xi}(r ; E)=(m r)^{\gamma} u_{+}-\xi(m r)^{-\gamma} u_{-}+O\left(r^{-\gamma+1}\right) .
$$

Тогда решение $V_{1}$ можно представить как

$$
V_{1}(r ; E) \equiv V_{\xi}=U_{\xi}(r ; E)+\frac{a+b}{2 s \gamma_{s}^{+}} \omega_{\xi}(E) U_{2}(r ; E)
$$

при

$$
\omega_{\xi}(E)=\operatorname{Wr}\left(U_{\xi}, V_{\xi}\right)=\omega(E)+\frac{2 s \gamma \xi}{a+b},
$$

где $\omega(E)$ определяется формулой (59).

Условие существования дискретного спектра энергий связанных состояний фермионов $\omega_{\xi}\left(E_{n \xi}\right)=0$ принимает вид

$$
\frac{\Gamma\left(2 \gamma_{s}^{+}\right) \Gamma\left(-\gamma_{s}^{+}+(1-s) / 2-(a E+m b) / \lambda\right)}{\Gamma\left(-2 \gamma_{s}^{+}\right) \Gamma\left(\gamma_{s}^{+}+(1-s) / 2-(a E+m b) / \lambda\right)} \frac{(2 \lambda)^{-2 \gamma_{s}^{+}}}{m^{-2 \gamma_{s}^{+}}} \frac{\left(1-m_{s}^{-}\right)}{\left(1-m_{s}^{+}\right)} \frac{2 s \gamma_{s}^{+}}{a+b}=-\frac{2 s \xi \gamma_{s}^{+}}{a+b} .
$$

Мы не получили формулу для дискретного спектра энергий связанных состояний фермионов в этой области в явном виде, однако можно показать, что спектр сгущается в точке $E=m$ и описывается асимптотической формулой (67), не зависящей от $\xi$ :

$$
\epsilon_{n \xi}=m-E_{n \xi}=\frac{m(a+b)^{2}}{2 n^{2}}, \quad n \rightarrow \infty .
$$

Анализ формулы (80) показывает, что $E$ существенно определяется величиной $a+b$ в области значений $E \cong m$ и $\sqrt{a^{2}-b^{2}}$ вблизи $E \cong 0$. Так как рассматриваемая квантовая система в основном состоянии может стать нестабильной при $\sqrt{a^{2}-b^{2}}>$ $1 / 2$, можно сказать, что скалярный потенциал стремится стабилизировать систему.

В работе [20] было показано, что в чисто кулоновском поле низший уровень энергии электрона может достичь границы нижнего континуума энергий уже в докритической области. Поведение низшего уровня энергии фермиона в рассматриваемом нами случае аналогично. Положим для простоты $b=0$. Тогда из формулы (80) в пределе $E=-m+\epsilon, \lambda=\sqrt{2 m \epsilon}, \epsilon \rightarrow+0$, получим следующее выражение для определения $\epsilon$ :

$$
\sqrt{\frac{\epsilon}{m}}\left[\xi(2 a)^{2 \gamma} a G-a-\frac{2 s \gamma(\nu+s \gamma)}{a}\right]=\sqrt{2} s \gamma\left[\xi(2 a)^{2 \gamma} G+1\right]+\sqrt{2} \nu\left[1-\xi(2 a)^{2 \gamma} G\right],
$$

откуда найдем точные значения $\xi(a, \gamma)$, при которых $\epsilon=0$ :

$$
\frac{1}{\xi}=(2 a)^{2 \gamma} \frac{\nu-s \gamma}{\nu+s \gamma} G, \quad G=\frac{\Gamma(-2 \gamma)}{\Gamma(2 \gamma)} .
$$

Кратко обсудим случай $\xi=\infty$. В этом случае любой дублет $F \in D_{\infty}$ при $r \rightarrow 0$ имеет асимптотическое поведение

$$
F(r)=c(m r)^{-\gamma} u_{-}+O\left(r^{1 / 2}\right) .
$$


В качестве дублетов $U(r ; E)$ и $V(r ; E)$ выберем

$$
U(r ; E)=U_{\infty}(r ; E)=U_{2}(r ; E), \quad U_{\infty}(r ; E)=(m r)^{-\gamma_{s}^{+}} u_{-}+O\left(r^{-\gamma_{s}^{+}+1}\right), \quad r \rightarrow 0,
$$

и

$$
V(r ; E)=\frac{2 s \gamma_{s}^{+}}{(a+b) \omega(E)} V_{1}(r ; E)=U_{2}(r ; E)-\frac{a+b}{2 s \gamma_{s}^{+}} \omega_{\infty}(E) U_{1}(r ; E)
$$

где

$$
\omega_{\infty}(E)=\operatorname{Wr}(U, V)=-\frac{4\left(\gamma_{s}^{+}\right)^{2}}{(a+b)^{2} \omega(E)} .
$$

Мы видим, что во второй докритической зарядовой области в случае $\xi=\infty$ спектр связанных состояний фермиона определяется полюсами функции $\omega(E)$.

4.3. Критические эффективные заряды $q=q_{\mathrm{c}}$. Эффективные заряды со

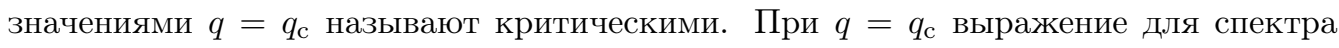
энергий (62) обращается в нуль, а при $q>q_{\text {c }}$ становится комплексным, что не позволяет интерпретировать это выражение как энергию. В этом случае

$$
q=q_{\mathrm{c}}=\nu \Longleftrightarrow \gamma_{s}^{+}=\gamma=0,
$$

и из формул (51)-(54) вытекает следующее асимптотическое поведение дублетов $F \in D^{*}$ при $r \rightarrow 0$ :

$$
F(r)=c_{1} u_{+}+c_{2} u_{-}^{0}+O\left(r^{1 / 2} \ln r\right) .
$$

Дублет с таким поведением не удовлетворяет условию (47), так как

$$
\left.\left(\bar{f}_{1} f_{2}-\bar{f}_{2} f_{1}\right)\right|_{r=0}=-\frac{s}{a+b}\left(\bar{c}_{1} c_{2}-\bar{c}_{2} c_{1}\right) .
$$

Эта формула аналогична формуле (70).

Следовательно, при $\gamma_{s}^{+}=0$ мы также имеем однопараметрическое $U(1)$-семейство самосопряженных операторов $h_{\xi}^{0}$ с областью определения $D_{\xi}^{0}$ :

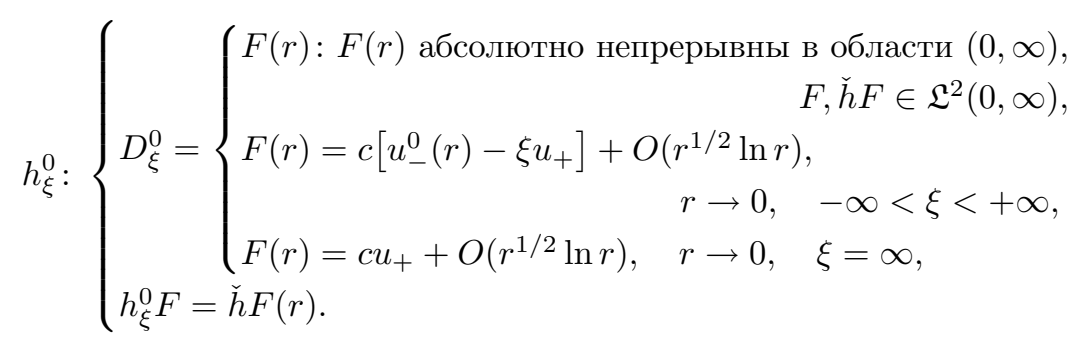

Значение $\xi=\infty$ отвечает эквивалентным случаям $\xi=+\infty$ и $\xi=-\infty$.

Обсудим случай $0<|\xi|<\infty$. В этом случае дублет $U_{\xi}^{0}(r ; E)$, определяющий направляющий функционал, является комбинацией

$$
U_{\xi}^{0}(r ; E)=U_{2}^{0}(r ; E)-\xi U_{1}(r ; E) .
$$

Можно проверить, что направляющий функционал в этом случае простой. При $r \rightarrow 0$

$$
U_{\xi}^{0}(r ; E)=u_{-}^{0}(r)-\xi u_{+}+O\left(r^{1 / 2} \ln r\right) .
$$


Вронскиан этих дублетов $\operatorname{Wr}\left(U_{\xi}^{0}, U_{1}\right)=s /(a+b)$. Решение $V_{1}^{0}$ удобно представить в виде

$$
V_{1}^{0}(r ; E)=U_{\xi}^{0}(r ; E)+\frac{a+b}{s} \omega_{\xi}^{0}(E) U_{1}(r ; E)
$$

где

$$
\omega_{\xi}^{0}(E)=\operatorname{Wr}\left(U_{\xi}^{0}, V_{1}^{0}\right)=\omega^{0}(E)+\frac{s \xi}{a+b}
$$

a

$$
\omega^{0}(E)=\frac{s}{a+b}\left\{\ln \left(\frac{2 \lambda}{m}\right)+\psi\left(-\frac{1-s}{2}-\frac{a E+m b}{\lambda}\right)-2 \psi(1)+\frac{s(a+b)(m+E)}{\nu^{2} \lambda+\nu(a+b)(m+E)}\right\} .
$$

Трансцендентное уравнение, определяющее дискретный спектр энергий фермионов $(|E|<m)$, в этом случае определяется нулями функции

$$
\omega_{\xi}^{0}(E)=\omega^{0}(E)+\frac{s \xi}{a+b} .
$$

Как и предыдущем случае, мы не смогли получить явную формулу для дискретного спектра энергий фермионов при $\gamma=0$, однако можно показать, что спектр сгущается в точке $E=m$ и описывается асимптотической формулой (81). Как и во второй докритической области, существуют значения $\xi$, при которых низший уровень энергии может достичь границы нижнего континуума энергий. Из формулы (80) в пределе $E=-m+\epsilon, \lambda=\sqrt{2 m \epsilon}, \epsilon \rightarrow+0$, нетрудно получить следующее приближенное выражение для определения $\epsilon$ и $\xi$ :

$$
\ln 2(a-b)-\ln z+\psi(z)-2 \psi(1)+\frac{s}{2 z}=-\xi, \quad z=(a-b) \sqrt{\frac{m}{2 \epsilon}} .
$$

Учитывая приближенное равенство $-\ln z+\psi(z) \cong 1 / 2 z, z \rightarrow \infty$, из выражения (94) найдем, что низший уровень энергии достигает границы нижнего континуума энергий при $-\xi=\ln 2(a-b)+2 \mathcal{C}$, где $\mathcal{C}=-\psi(1)=0.57721-$ постоянная Эйлера [29].

Обсудим теперь случай $\xi=\infty$, когда для любого дублета $F \in D_{\infty}$

$$
F(r)=c u_{+}+O\left(r^{1 / 2} \ln r\right), \quad r \rightarrow 0 .
$$

В качестве дублетов $U(r ; E)$ и $V(r ; E)$ выберем

$$
\begin{gathered}
U(r ; E)=U_{\infty}^{0}(r ; E)=U_{1}(r ; E), \quad U_{\infty}^{0}(r ; E)=c u_{+}+O(r), \quad r \rightarrow 0, \\
V(r ; E)=\frac{s}{(a+b) \omega^{0}(E)} V_{1}^{0}(r ; E)=U_{1}(r ; E)-\frac{a+b}{s} \omega_{\infty}^{0}(E) U_{2}^{0}(r ; E),
\end{gathered}
$$

где

$$
\omega_{\infty}^{0}(E)=\operatorname{Wr}(U, V)=-\frac{1}{(a+b)^{2} \omega^{0}(E)} .
$$

В этой зарядовой области спектр связанных состояний фермиона при $\xi=\infty$ определяется полюсами функции $\omega^{0}(E)$; в явном виде он задается формулой $(62)$, в которой нужно положить $\gamma_{s}^{+}=0$. Полагая в выражении $(66)(\beta+1 / 2)^{2}+b^{2}=a^{2}$, найдем энергию фермиона в низшем состоянии с $s=1$ для $\mu>0$ или с $s=-1$ для $\mu<0$ в виде $E_{\infty}^{s}=-m b / a$. 


\section{5. ОБЛАСТЬ СВЕРХКРИТИЧЕСКИХ ЭФФЕКТИВНЫХ ЗАРЯДОВ}

В сверхкритической области

$$
q>q_{\mathrm{c}}=\nu=l+\mu+\frac{s}{2} \Longleftrightarrow \gamma_{s}^{+}=i \sigma, \quad \sigma=\sqrt{\left(a^{2}-b^{2}\right)-\nu^{2}}>0,
$$

любой дублет $F \in D^{*}$ при $r \rightarrow 0$ должен вести себя как

$$
F(r)=c_{1}(m r)^{i \sigma} u_{+}+c_{2}(m r)^{-i \sigma} u_{-}+O\left(r^{1 / 2}\right), \quad u_{ \pm}=\left(\begin{array}{c}
( \pm i s \sigma+\nu) /(a+b) \\
1
\end{array}\right) .
$$

Тогда левая часть выражения (47) принимает вид

$$
\left.\left(\bar{f}_{1} f_{2}-\bar{f}_{2} f_{1}\right)\right|_{r=0}=-\frac{2 i s \sigma}{a+b}\left(\left|c_{1}\right|^{2}-\left|c_{2}\right|^{2}\right) \text {. }
$$

Таким образом, и при $\gamma_{s}^{+}=i \sigma$ мы имеем однопараметрическое $U(1)$-семейство самосопряженных операторов $h_{\theta}, 0 \leqslant \theta \leqslant \pi, 0 \sim \pi$, удовлетворяющих соотношению (97), с областью определения $D_{\theta}$ :

$$
h_{\theta}:\left\{\begin{aligned}
D_{\theta}=\left\{\begin{array}{l}
F(r): F(r) \text { абсолютно непрерывны в области }(0, \infty), \\
F(r)=c\left[e^{i \theta}(m r)^{i \sigma} u_{+}+e^{-i \theta}(m r)^{-i \sigma} u_{-}\right]+O\left(r^{1 / 2}\right), \\
0 \leqslant \theta \leqslant \pi, \quad 0 \sim \pi,
\end{array}\right. \\
h_{\theta} F=\check{h} F(r),
\end{aligned}\right.
$$

где $c$ - комплексная константа.

Дублет, определяющий направляющий функционал, имеет вид

$$
U_{\theta}(r ; E)=e^{i \theta} U_{1}(r ; E)+e^{-i \theta} U_{2}(r ; E),
$$

где $U_{1}(r ; E), U_{2}(r ; E)$ являются дублетами $(27)-(34)$ с $\gamma_{s}^{+}=i \sigma$. Следовательно, асимптотическое поведение дублета имеет вид

$$
U_{\theta}(r ; E)=e^{i \theta}(m r)^{i \sigma} u_{+}+e^{-i \theta}(m r)^{-i \sigma} u_{-}, \quad r \rightarrow 0 .
$$

Можно проверить, что направляющий функционал, как и в предыдущих случаях, простой. Функцию Грина оператора $h_{\theta}$ можно сконструировать, используя дублеты $U_{\theta}(r ; E)$ и $V_{\theta}(r ; E)$ :

$$
\begin{gathered}
V_{\theta}(r ; E)=\frac{2}{e^{-i \theta}+e^{i \theta} \widetilde{\omega}(E)} V_{1}(r ; E)=U_{\theta}(r ; E)-\frac{a+b}{4 s \sigma} \omega_{\theta}(E) \widetilde{U}_{\theta}(r ; E), \\
\widetilde{U}_{\theta}=\frac{1}{i}\left[e^{i \theta} U_{1}(r ; E)-e^{-i \theta} U_{2}(r ; E)\right], \\
\widetilde{\omega}=\frac{a+b}{2 s i \sigma} \omega(E), \quad \omega_{\theta}(E)=\operatorname{Wr}\left(U_{\theta}, V_{\theta}\right)=-\frac{4 i s \sigma}{a+b} \frac{1-\widetilde{\omega}(E) e^{2 i \theta}}{1+\widetilde{\omega}(E) e^{2 i \theta}},
\end{gathered}
$$

где $V_{1}(r ; E)$ и $\omega(E)$ заданы формулами $(35),(36)$ при $\gamma_{s}^{+}=i \sigma$. Конечное выражение для производной спектральной функции гамильтониана $h_{\theta}$ дается формулой

$$
\frac{d \sigma_{\theta}(E)}{d E}=\frac{1}{\pi} \lim _{\epsilon \rightarrow 0} \operatorname{Im} \frac{1}{\omega_{\theta}(E+i \epsilon)}
$$


В этой зарядовой области спектральная функция определяется обобщенной функцией $F_{\theta}(E)=\lim _{\epsilon \rightarrow 0} \omega_{\theta}^{-1}(E+i \epsilon) ;$ в точках, в которых функция $\omega_{\theta}(E)=\lim _{\epsilon \rightarrow 0} \omega_{\theta}(E+i \epsilon)$ отлична от нуля, $F_{\theta}(E)=1 / \omega_{\theta}(E)$.

Для связанных состояний $|E|<m$ имеем

$$
\widetilde{\omega}(E)=\frac{\Gamma(2 i \sigma)}{\Gamma(-2 i \sigma)} \frac{\Gamma(-i \sigma+(1-s) / 2-(a E+m b) / \lambda)}{\Gamma(i \sigma+(1-s) / 2-(a E+m b) / \lambda)} \frac{(2 \lambda)^{-2 i \sigma}}{m^{-2 i \sigma}} \frac{1-m_{s}^{-}}{1-m_{s}^{+}} \equiv e^{-2 i \Omega(E)} .
$$

Пусть далее по определению

$$
\begin{aligned}
\phi_{E} & =\frac{1}{2 i}\left[\ln \Gamma\left(\frac{1-s}{2}-\frac{a E+m b}{\lambda}-i \sigma\right)-\ln \Gamma\left(\frac{1-s}{2}-\frac{a E+m b}{\lambda}+i \sigma\right)\right], \\
\phi_{s} & =\frac{1}{2 i}\left[\ln \left(\nu+i s \sigma+\frac{(E+m)(a+b)}{\lambda}\right)-\ln \left(\nu-i s \sigma+\frac{(E+m)(a+b)}{\lambda}\right)\right], \\
\phi_{\sigma} & =\frac{\ln \Gamma(2 i \sigma)-\ln \Gamma(-2 i \sigma)}{2 i} .
\end{aligned}
$$

Тогда функция

$$
\omega_{\Omega}(E)=\frac{4 s \sigma}{a+b} \operatorname{tg}[\Omega(E)-\theta]
$$

вещественна, а спектр самосопряженного гамильтониана $\left(h_{\theta}\right)$ неявно определяется уравнением

$$
\Omega(E)-\theta \equiv \sigma \ln \frac{2 \lambda}{m}-\phi_{\sigma}-\phi_{E}-\phi_{s}-\theta=n \pi, \quad n=0, \pm 1, \pm 2, \ldots
$$

Следует подчеркнуть, что уравнение (106) неявно определяет собственные значения энергии фермиона в связанных состояниях в области $m>E \geqslant-m$. Анализ полученной формулы показывает, что точка $E=m$ является точкой сгущения спектра, а при $n \gg 1$ спектр описывается правой частью асимптотической формулы (81); что в интервале $-m \leqslant E<0$ число дискретных уровней энергии конечно. Нелишне также отметить, что вблизи границы нижнего континуума, т. е. при $E=-m+\epsilon, \epsilon>0$, формула (106) имеет ту же структуру, что и формула для спектра энергий электрона в сильном (обрезанном на малом расстоянии $R$ ) кулоновском и АБ-потенциалах в $2+1$ измерениях вблизи $-m$ (см. работу [19]).

Для практического использования формулы (106) с помощью разложения (см. монографию [30], с. 174)

$$
\Gamma(x+i y)=|\Gamma(x+i y)| e^{i \eta}, \quad \eta=y\left[-\mathcal{C}+\sum_{n=1}^{\infty}\left(\frac{1}{n}-\frac{1}{y} \operatorname{arctg} \frac{y}{x+n-1}\right)\right],
$$

можно получить для аргументов гамма-функций удобные для анализа явные выражения. Если эффективный заряд $\sqrt{a^{2}-b^{2}}$ слегка сверхкритический, т. е. $0<\sigma \ll 1$, то вблизи границы нижнего континуума $E=-m+\epsilon, \epsilon>0$, уравнение (106) в пределах $\epsilon \rightarrow+0,0<\sigma \ll 1, \lambda=\sqrt{2 m \epsilon}$, приводится к виду

$$
\theta-\frac{\pi}{2}+\sigma \ln \frac{2}{a-b}+\operatorname{arctg} \frac{s \sigma}{\nu+(a+b) \sqrt{\epsilon / 2 m}}-\sigma \mathcal{C}=-n \pi
$$


Из этой формулы следует, что в пределе $E=-m+\epsilon \rightarrow+0$ при $0<\sigma \ll 1$ допустимо только одно значение $n=0$, т. е. низший уровень энергии достигает границы нижнего континуума энергий при

$$
\theta=\frac{\pi}{2}-\sigma \ln \frac{2}{a-b}-\operatorname{arctg} \frac{s \sigma}{\nu+(a+b)}+\sigma \mathcal{C} .
$$

\section{6. О СОБСТВЕННЫХ ФУНКЦИЯХ СВЯЗАННЫХ СОСТОЯНИЙ}

Обсудим вопрос о собственных состояниях (функциях) самосопряженного гамильтониана, относящихся к дискретному спектру. Эти собственные функции должны принадлежать гильбертову пространству $\mathfrak{L}^{2}(0, \infty)$. В области энергий $|E|<m$ при малых эффективных зарядах дублеты, квадратично-интегрируемые на бесконечности, можно представить в виде

$$
F(r)=A V_{1}(r ; E)=A\left[U_{1}(r ; E)+\frac{a+b}{2 s \gamma_{s}^{+}} \omega(E) U_{2}(r ; E)\right],
$$

где $A$ - постоянная, а $\omega(E)$ определяется формулой (59). Очевидно, эти решения интегрируемы с квадратом в источнике (и, следовательно, на всей полуоси) только при выполнении условия $\omega(E)=0$.

Во второй докритической области квадратично-интегрируемые на всей полуоси дублеты $($ с $|E|<m)$ найдены в виде

$$
F(r)=A V_{1}(r ; E)=A\left[U_{\xi}(r ; E)+\frac{a+b}{2 s \gamma_{s}^{+}} \omega_{\xi}(E) U_{2}(r ; E)\right],
$$

где $A$ - постоянная, а $\omega_{\xi}(E)$ определяется формулой (79). Однако эти решения удовлетворяют самосопряженным граничным условиям в нуле (75), только если $\omega_{\xi}(E)=0$.

Для критических зарядов квадратично-интегрируемые на всей полуоси дублеты $($ с $|E|<m)$ имеют вид

$$
F(r)=A V_{1}^{0}(r ; E)=A\left[U_{\xi}(r ; E)+\frac{a+b}{s} \omega_{\xi}^{0}(E) U_{1}(r ; E)\right],
$$

где $A$ - постоянная, а $\omega_{\xi}^{0}(E)$ определяется формулой (91). Однако найденные дублеты удовлетворяют самосопряженным граничным условиям в нуле (87), только если $\omega_{\xi}^{0}(E)=0$.

Аналогично можно построить собственные функции самосопряженного гамильтониана при сверхкритических зарядах. В этом случае квадратично-интегрируемые (в интервале энергий $|E|<m$ ) на всей полуоси решения имеют вид

$$
F(r)=A V_{\theta}(r ; E)=A\left[U_{\theta}(r ; E)-\frac{a+b}{4 s \sigma} \omega_{\theta}(E) \widetilde{U}_{\theta}(r ; E)\right],
$$

где $A$ - постоянная, а $\omega_{\theta}(E)$ и дублеты $V_{\theta}(r ; E), U_{\theta}(r ; E), \widetilde{U}_{\theta}(r ; E)$ определяются формулами (99)-(101). Если положить $\omega_{\theta}(E)=0$, то эти решения будут удовлетворять асимптотическим самосопряженным граничным условиям (98).

Нелишне подчеркнуть, что приведенные выше решения являются собственными функциями самосопряженного радиального гамильтониана в соответствующих областях значений параметров лишь при выполнении асимптотических самосопряженных граничных условий. Только такие решения отвечают состояниям дискретного спектра самосопряженного радиального гамильтониана. 


\section{7. НЕПРЕРЫВНЫЙ СПЕКТР}

В заключение кратко обсудим вопрос об областях непрерывного спектра самосопряженного радиального гамильтониана $|E| \geqslant m$. Прямая проверка показывает, что в зарядовой области $\gamma_{s}^{+}>1 / 2$ функция $\omega(E)(36)$ непрерывна, отлична от нуля и имеет комплексные значения. Следовательно, спектральная функция $\sigma(E)$ в области энергий $|E| \geqslant m$ абсолютно непрерывна и

$$
\begin{aligned}
\frac{d \sigma(E)}{d E}= & \frac{1}{\pi} \operatorname{Im} \frac{1}{\omega(E)}, \\
\omega(E)= & \frac{\Gamma\left(2 \gamma_{s}^{+}\right) \Gamma\left(-\gamma_{s}^{+}+(1-s) / 2+(a|E|+m b) / i e k\right)}{\Gamma\left(-2 \gamma_{s}^{+}\right) \Gamma\left(\gamma_{s}^{+}+(1-s) / 2+(a|E|+m b) / i e k\right)} \times \\
& \times \frac{(-i e k)^{-2 \gamma_{s}^{+}}}{m^{-2 \gamma_{s}^{+}}} \frac{2 s \gamma_{s}^{+}}{a+b} \frac{\nu-(a+b)(m+E) / i e k+s \gamma_{s}^{+}}{\nu-(a+b)(m+E) / i e k-s \gamma_{s}^{+}}, \\
e= & \frac{E}{|E|}, \quad k=\sqrt{E^{2}-m^{2}} .
\end{aligned}
$$

В области $|E| \geqslant m$ функция $\omega_{\xi}(E)$ для $0<\gamma_{s}^{+}<1 / 2$ непрерывна, отлична от нуля и комплексна, как и $\omega(E)(108)$. Следовательно, спектральная функция $\sigma_{\xi}(E)$ в области $|E| \geqslant m$ при $\xi \neq 0$ абсолютно непрерывна и

$$
\frac{d \sigma_{\xi}(E)}{d E}=\frac{1}{\pi} \operatorname{Im} \frac{1}{\omega(E)+2 s \gamma \xi /(a+b)},
$$

что является аналогом формулы $(108)$ с заменой $\omega(E)$ на $\omega_{\xi}(E)$.

В области $|E| \geqslant m$ функция $\omega^{0}(E)$ для $\gamma_{s}^{+}=0$ дается выражением

$$
\begin{aligned}
\omega^{0}(E)= & \frac{s}{a+b}\left[\ln \left(2 e^{-i e \pi / 2} \frac{k}{m}\right)+\psi\left(-\frac{1-s}{2}+\frac{a|E|+m b}{i e k}\right)-\right. \\
& \left.-2 \psi(1)+\frac{s(a+b)(m+E)}{-i e k \nu^{2}+\nu(a+b)(m+E)}\right] .
\end{aligned}
$$

Она непрерывна, отлична от нуля и комплексна, поэтому спектральная функция $\sigma_{\xi}^{0}(E)$ в области $|E| \geqslant m$ при $\xi \neq 0$ абсолютно непрерывна и

$$
\frac{d \sigma_{\xi}^{0}(E)}{d E}=\frac{1}{\pi} \operatorname{Im} \frac{1}{\omega^{0}(E)-s \xi /(a+b)} .
$$

В области $|E| \geqslant m$ функция $\omega_{\theta}(E)$ для $\gamma_{s}^{+}=i \sigma$ дается выражением (101), в котором в $\widetilde{\omega}(E)$ нужно положить $\lambda=-i k$. Она непрерывна, отлична от нуля и комплексна, поэтому спектральная функция $\sigma_{\theta}(E)$ в области $|E| \geqslant m$ абсолютно непрерывна и

$$
\frac{d \sigma_{\theta}(E)}{d E}=\frac{1}{\pi} \operatorname{Im} \frac{1}{\omega_{\theta}(E)} .
$$

Собственные функции самосопряженного радиального гамильтониана, относящиеся к непрерывному спектру энергий, должны допускать “нормировку на $\delta$-функцию”.

Благодарности. В.Р. Халилов благодарен И. В. Тютину за многочисленные ценные замечания и полезные рекомендации. 


\section{Список литературы}

[1] A. M. J. Schakel, G. W. Semenoff, Phys. Rev. Lett., 66:20 (1991), 2653-2656.

[2] Y. Aharonov, D. Bohm, Phys. Rev., 115:3 (1959), 485-491.

[3] Р. Прендж, С. Гирвин (ред.), Квантовый эфбект Холла, Мир, М., 1989.

[4] F. Wilczek, Fractional Statistics and Anyon Superconductivity, World Scientific, Teaneck, NJ, 1990.

[5] A. Neagu, A. M. J. Schakel, Phys. Rev. D, 48:4 (1993), 1785-1791, arXiv: hep-th/9306092.

[6] M. G. Alford, F. Wilczek, Phys. Rev. Lett., 62:10 (1989), 1071-1074.

[7] Ph. De Sousa Gerbert, Phys. Rev. D, 40:4 (1989), 1346-1349.

[8] M. G. Alford, J. March-Pussel, F. Wilczek, Nucl. Phys. B, 328:1 (1989), 140-158.

[9] K. S. Novoselov A. K. Geim, S. V. Morozov, D. Jiang, M. I. Katsnelson, I. V. Grigorieva, S. V. Dubonos, A. A. Firsov, Nature, 438:7065 (2005), 197-200, arXiv: cond-mat/0509330.

[10] Z. Jiang, Y. Zhang, H. L. Stormer, P. Kim, Phys. Rev. Lett., 99:10 (2007), 106802, 4 pp., arXiv: 0705.1102.

[11] I. F. Herbut, Phys. Rev. Lett., 104:6 (2010), 066404, 4 pp., arXiv: 0909.4231.

[12] И.В. Тютин, Рассеяние электронов соленоидом, препринт № 27, ФИАН, М., 1974, arXiv: 0801.2167.

[13] Я. Б. Зельдович, В. С. Попов, УФН, 105:3-4 (1971), 403-440.

[14] А.Б. Мигдал, Фермионы и бозоны в силъных полях, Наука, М., 1978.

[15] J. Rafelski, L. P. Fulcher, A. Klein, Phys. Rep., 38:5 (1978), 227-361.

[16] M. Soffel, B. Müller, W. Greiner, Phys. Rep. C, 85:2 (1982), 51-122.

[17] W. Greiner, J. Reinhardt, Quantum Electrodynamics, Springer, Berlin, 2009.

[18] В. Р. Халилов, ТМФ, 116:2 (1998), 277-287.

[19] В. Р. Халилов, ТМФ, 158:2 (2009), 250-262.

[20] Б. Л. Воронов, Д. М. Гитман, И. В. Тютин, ТМФ, 150:1 (2007), 41-84.

[21] D. M. Gitman, A. A. Smirnov, I. V. Tyutin, B. L. Voronov, Self-adjoint Schrödinger and Dirac operators with Aharonov-Bohm and magnetic-solenoid fields, arXiv: 0911.0946.

[22] М.А. Наймарк, Линейные дифференциальные операторы, Наука, М., 1969.

[23] С. Г. Крейн (ред.), Функииональный анализ, Наука, М., 1972.

[24] C. R. Hagen, Phys. Rev. Lett., 64:5 (1990), 503-506.

[25] V.R. Khalilov, C.-L. Ho, Ann. Phys., 323:5 (2008), 1280-1293, arXiv: 0708.3131.

[26] V.R. Khalilov, Phys. Rev. A, 71:1 (2005), 012105, 6 pp., arXiv: quant-ph/0406033.

[27] B. L. Voronov, D. M. Gitman, I. V. Tyutin, Self-adjoint differential operators associated with self-adjoint differential expressions, arXiv: quant-ph/0603187.

[28] В.Б. Берестецкий, Е.М. Лифшиц, Л. П. Питаевский, Теоретическая физика, т. 4: Квантовая электродинамика, Наука, М., 1980.

[29] И. С. Градштейн, И.М. Рыжик, Таблицы интегралов, сумм, рядов и произведений, Физматлит, М., 1971.

[30] З. Флюгге, Задачи по квантовой механике, Мир, М., 1974.

Поступила в редакцию 8.12.2010, после доработки 9.03.2011 